\title{
Improved Targeting through Collaborative Decision-Making and Brain Computer Interfaces
}

\author{
Adrian Stoica \\ David F. Barrero \\ Klaus Mc Donald-Maier \\ Jet Propulsion Laboratory \\ Departamento de Automática \\ Universidad de Alcalá \\ School of Computer Science and Electronic Engineering \\ California Institute of Technology \\ Pasadena, CA, USA \\ Alcalá de Henares, Madrid, Spain \\ Email: david@aut.uah.es \\ University of Essex \\ Colchester, UK \\ Email: adrian.stoica@jpl.nasa.gov \\ Email: kdm@essex.ac.uk
}

\begin{abstract}
This paper reports a firs step toward a braincomputer interface (BCI) for collaborative targeting. Specificall , we explore, from a broad perspective, how the collaboration of a group of people can increase the performance on a simple target identificatio task. To this end, we requested a group of people to identify the location and color of a sequence of targets appearing on the screen and measured the time and accuracy of the response. The individual results are compared to a collective identificatio result determined by simple majority voting, with random choice in case of drawn. The results are promising, as the identificatio becomes significantl more reliable even with this simple voting and a small number of people (either odd or even number) involved in the decision. In addition, the paper briefl analyzes the role of brain-computer interfaces in collaborative targeting, extending the targeting task by using a BCI instead of a mechanical response.
\end{abstract}

\section{INTRODUCTION}

Brain computer interfaces $(\mathrm{BCI})[1],[2]$ provide an alternative to classical interfaces, such as the ones based on mechanical interactions. BCI, instead, analyzes the neuronal activity through a set of electrodes placed on the head. However, it has to deal with some notable difficulties due to a weak signal and the presence of large noise generated by the body and the environment. In order to overcome these limitations, as well as for opening avenues to address more complex problems by a division of focus of attention, memory and overall effort among multiple individuals, a set of recent publications propose an extension of $\mathrm{BCI}$ named Collaborative BCI (CBCI) [3], [4], [5], [6].

$\mathrm{CBCI}$ tries to overcome the problem of $\mathrm{BCI}$ by aggregating the signals reflectin the activity of several brains, opening a new set of applications, albeit a new set of difficulties In addition, CBCI exploits collaboration. It is generally accepted that collaboration increases the quality of decision in comparison to individual decisions [7]. Early indications to these trace back, for example, to the work of Galton, who in an early paper [8], asked a group of people to guess the weight of a person, and showed that the average value of the guesses was very close to the real weight. A more detailed discussion about this topic can be found in [9].

In this paper we analyze one particular application, targeting, and explore the potential of CBCI to address this problem. The motivation behind this study is an interest for developing
BCI to allow a rapid action of humans controlling, with different levels of involvement, a deployed platform, which can be a sub-system on an unmanned aerial vehicle (UAV), or the UAV itself. For space unmanned systems working under human command, a number of potential applications of BCI have been proposed [10], [11], such as the control of a future spacecraft [12]. Another possible application is an early-alarm system for manned space-missions which, in case of an emergency, activates an emergency response based on the astronauts brain-responses. In this scenario the time and accuracy of the decision making is critical, so using a CBCI would bring shorter and more reliable decision making [13], [1]. The particular application of target identificatio is extremely relevant to games (in this case in particular collaborative games), and of course, to military scenarios.

This paper reports an exploratory study to analyze the feasibility of CBCI to address the type of previously described applications. We aim, firstly to verify experimentally whether collective targeting outperforms individual identificatio accuracy. As a second goal we investigate the relationship between time and collaboration in order to determine if it is possible to reduce the decision time by using a collective identification The third objective is, based on the gathered data, to suggest better voting systems in terms of accuracy and time. Finally, we try to substitute the input by keystrokes with EEG signals.

The paper is structured as follows. Firstly, we describe the experiment and the voting scheme that we applied. Then we report the experimental results from the perspective of the accuracy and section IV introduces the decision time. A more detailed description of the results per target follows in section V. SectionVI introduces a brief EEG analysis in order to construct a BCI for target identification The paper finis es outlining some conclusions and future work.

\section{EXPERIMENTAL DESIGN}

\section{A. Experimental procedure}

A group of six people was requested to identify the location and the color of a target sequence on a screen. The targets are colored squares that appear, one at a time, on a $2 \times 3$ matrix (six potential locations) and have one of possible three colors: 


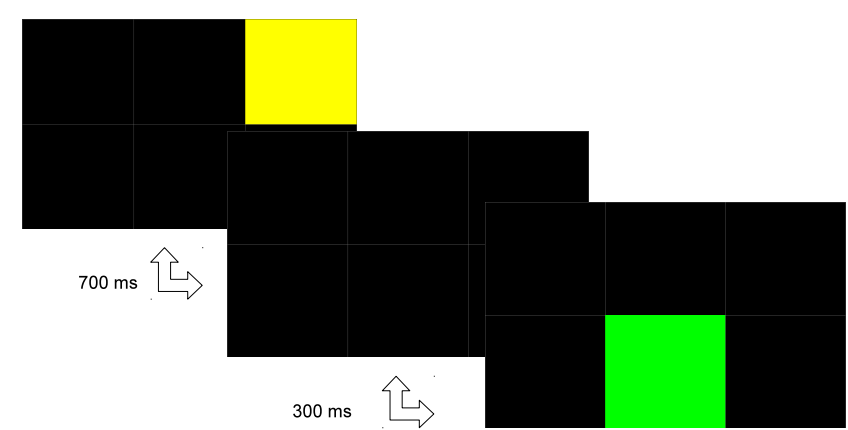

Fig. 1. Screenshot that shows the sequence target-pause-target. The subjects had to identify the location and color of a sequence with 30 targets.

TABLE I

SUMMARY OF THE MAIN EXPERIMENTAL PARAMETERS.

\begin{tabular}{|c|l|}
\hline Parameter & Value and description \\
\hline Subjects & 6, ages between 27 and 43,5 males and 1 female \\
Trials & One serie with 30 trials \\
Stimuli location & Matrix $3 \times 2$ \\
Stimuli color & Green, yellow and red \\
Stimuli time & 700 milliseconds \\
Stimuli Pause time & 300 milliseconds \\
\hline
\end{tabular}

green, yellow and red ${ }^{1}$. The screen showed the targets in a sequence, f rst, it showed the target along 700 milliseconds, then there was a pause of 300 milliseconds (see Fig. 1). After the pause, the screen showed the next target. The sequence shown to the subjects contained 30 targets generated randomly; this sequence remained the same for all the subjects to expose them to the same task diff culty.

The subjects provided the answers mechanically with a computer keyboard. Each location and color had an associated key in the keyboard. The subjects could provide the location and color in the order they preferred, but once they provided an answer, they could not change it. The assignment of keys to locations and colors was explained to each subject before performing the experiment. We observed that the results, in terms of accuracy and decision time, were very low for subjects who took the test without a prior training. As

a consequence each subject was trained with three series of 15 targets before gathering the data for processing.

Given that the objective is to determine how a collective identif cation of the target can improve the accuracy and decision time, we set the target times hard enough to make most subject fail about $70 \%$ of the trials. The timing was set on the basis of the results of an exploratory experiment. Table I summarizes the main parameters in the experiment.

For each subject in the experiment, we recorded the location and color that they identif ed, as well as the ground truth. In order to add the time to the analysis, we also recorded, for

\footnotetext{
${ }^{1}$ We also tried this experiment with bigger matrices and higher number of targets, however, the keyboard turned out to be a bad input device in those conditions because of the mapping between locations/colors and keys. We also tried with a mouse, yet the mouse movement required too much time and had an unacceptable inf uence on the time measurements. Finally we decided to use a small matrix with three colors and a keyboard as input device.
}

each target, the time elapsed from its visualization to the $\mathrm{frst}$ keystroke, which is referred in the following as the reaction time (RT).

\section{B. Votation mechanism}

The voting mechanism is simple majority. We consider the individual responses of the subjects as votes, and the collective response is assigned to be the identif cation that gathers most votes. In this way if, for instance, two subjects identify the target as red, and one subject as green, the collective response is red. This study carried out an experiment with two identif cation tasks: location and color. Despite the are reasons to think that they can have some interactions, we have considered them as independent tasks and, therefore, we made two votings for each subject and target, one for location and the another one for color. In this way we keep the decision making simple, providing a baseline to compare future works. When a subject was unable to provide an answer in the given time, we consider that he made a blank vote.

Majority systems have to deal with draws. In our experiments, we choose a naïve draw resolution mechanism that consists on randomly selecting one of the disputed identif cation. Draws happen more when there are few subjects and when the number of subjects is even; the worst scenario from this point of view is when there are only two voting subjects, in which case any disagreement results in a draw. Another issue is when there are no valid votes, in this case, the system is unable to determine the result because it lacks any information to guide its decision, and then we say that the response is empty.

We intend to explore more sophisticated voting mechanisms, which might, for example, learn from prior experience and favor the response of subjects who did particularly well for the specif c context, or vice-versa, weight less the responses from users who consistently made specif c mistakes; color and location may also be correlated. At this stage we only implemented a simple voting mechanism that considers location and color tasks as independent. This simple voting system has nonetheless proven successful, and serves as reference to compare future voting mechanisms.

\section{ACCURACY OF COLLECTIVE TARGET IDENTIFICATION}

The individual accuracy (ratio of the correct identif cations) and RT provides a basis to compare the collective results. To this end, Table II reports the individual accuracy and number of empty responses of all the subjects involved in the experiment.

Subject six is the best scoring individual in the location task, with an accuracy 0.9; analogously, the most accurate individual in color identif cation is subject three, with 0.93 . It turns out that subject six is also the one with the lowest mean RT, which is $592 \mathrm{~ms}$. The slowest subject was subject two, whose median RT is $752 \mathrm{~ms}$. RT is discussed in detail in section IV.

Figure 2 visualizes the relationship between the number of subjects involved in the collective identif cation and the accuracy. It reveals that the accuracy increases when more people are involved in the decision. To compute the accuracy 
TABLE II

INDIVIDUAL ACCURACY IDENTIFYING THE TARGET LOCATION AND COLOR, EACH SUBJECT WAS STIMULATED WITH 30 TARGETS. THE TABLE ALSO REPORTS THE NUMBER OF EMPTY RESPONSES (NO VALID VOTE).

\begin{tabular}{|l|l|l|l|l|l|}
\hline & \multicolumn{2}{|c|}{ Location } & \multicolumn{2}{c|}{ Color } & Mean RT (ms) \\
\hline & Accuracy & Empty & Accuracy & Empty & \\
\hline Subject 1 & 0.47 & 12 & 0.67 & 6 & $647 \pm 102$ \\
Subject 2 & 0.6 & 10 & 0.5 & 10 & $752 \pm 95$ \\
Subject 3 & 0.67 & 7 & 0.93 & 0 & $728 \pm 77$ \\
Subject 4 & 0.83 & 4 & 0.23 & 23 & $723 \pm 68$ \\
Subject 5 & 0.8 & 5 & 0.87 & 0 & $674 \pm 124$ \\
Subject 6 & 0.9 & 2 & 0.77 & 5 & $592 \pm 85$ \\
\hline
\end{tabular}
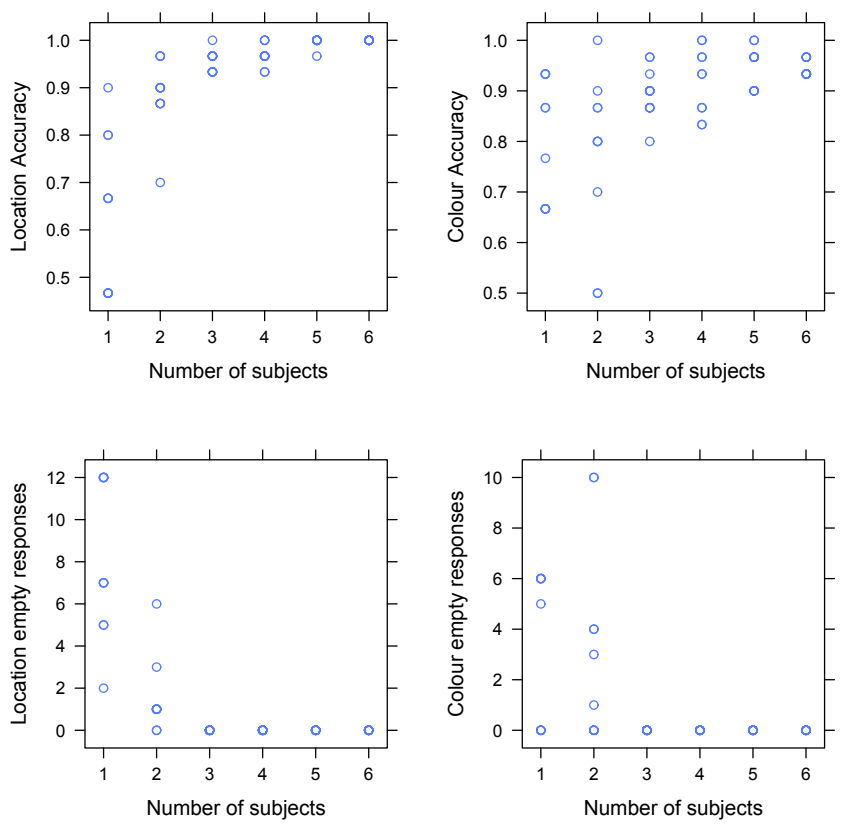

Fig. 2. Accuracy of location (left) and color (right) identif cation involving a varying number of subjects. With only one subject the voting gives the same result that considering the subject alone. Each point in the diagram represents the result of the collective decision (or of a single individual in case of a single participant). For each number of subjects we sampled ten times and computed the accuracy and number of empty responses.

shown in Fig. 2 we took $n \in\{1,2,3,4,5,6\}$ random subjects, computed the accuracy with the 30 targets and averaged the value, repeating this procedure 10 times. We should point out that when $n=1$ the target identif cation is individual, there is no collective decision from multiple individuals and, as a consequence, the result is the same as shown in Table II.

The most interesting fact in Fig. 2 is the accuracy increase with the number of subjects. Related to this, the number of empty responses decreases with the number of subjects as well and hence both perspectives indicate a clear benef $t$ of collective decision making. However, the increase of the decision quality was bounded. Adding more subjects increased the accuracy up to a point placed around three people. From that point, for this experiment, adding new subjects had a limited impact.

Of course, accuracy and empty responses are closely related to each other: An empty response means that the subject failed identifying a target, and therefore it reduces the accuracy. It is clear when comparing the location accuracy and empty responses in Fig. 2; the former reduces the empty responses dramatically with three subjects, meaning that there is, at least, one valid response, but the accuracy is not one, meaning that there are incorrect identif cations. As the number of subjects increases, the accuracy also increases. Interestingly, color identif cation has a slightly different behavior. As in location identif cation, the number of empty answers went to zero with three subjects, but the increase of accuracy is much more modest.

Table II shows that the best subject identifying the location (subject six) achieved an accuracy of 0.9 , while the best one in color identif cation is subject three with 0.93 . It is worth to compare these best individuals with the collective identif cation. With two subjects, the mean accuracy increases and its variability drops as well; the accuracy is similar to the accuracy of the best single individual in average terms. When the number of subjects increases to three, the resulting accuracy always improves over the best individual.

Color identif cation has a slightly different behavior. Increasing the number of subjects has a less evident benef $t$. It reduces the variability slowly, so, the increase of the mean accuracy with the number of subjects is less evident. Actually, in this case, the best individual (subject five) almost always has a better accuracy (0.93) then collective identif cation, which requires six subjects to equal the best individual. The poor performance of subject four in color identif cation (0.23) and the low number of subjects may explain this result. When subject four is sampled to compute the accuracy, he reduces signif cantly the color identif cation accuracy. In a more intelligent voting scheme this information can be used to improve the result after learning performance models for users, and not including them in specif $\mathrm{c}$ aspects of decision where they consistently sub-perform.

The accuracy improvement when two subjects are involved, in comparison to one, is an interesting, unexpected result. With two subjects, in case of disagreement, the decision is taken at random with almost no information, and this can happen often. Under these conditions one could expect poor results, but, on the contrary, the increase of the accuracy is notable, more if we consider the naïve voting mechanism. To explain this, we can take the opposite point of view - even though the subjects disagree in what the correct identif cation is, at least they agree what identif cations are incorrect. Subject A might say that the target is green, and subject B that it is yellow, but in this case both agree the target is not red. This information is considered implicitly by the draw resolution mechanism adopted, and is the cause for accuracy improvement.

The conclusion in this section is that the collective decision outperformed best individual decision in the location identif cation task. However, the effect of the collaboration in color identif cation is less evident, probably due to the presence of a poor performance subject in the experiment. The accuracy is important in almost any application we can envision, however, it only provides a partial view of the reality. To complete 


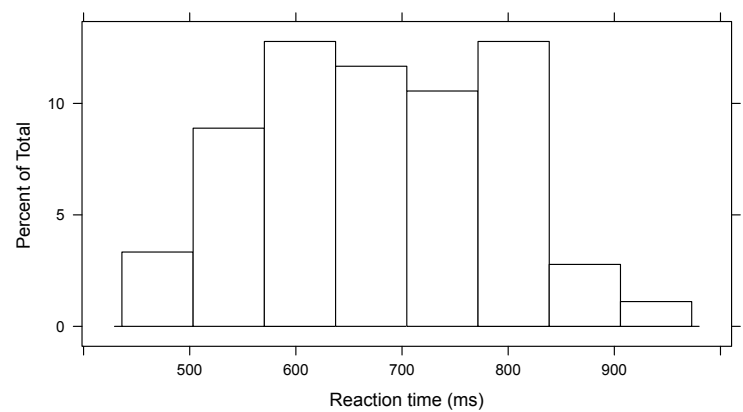

Fig. 3. Histogram of the reaction time.

our understanding, we have to include the study of the time needed for target identif cation by a collective decision.

\section{TIME TO IDENTIFY THE TARGET}

The decision time plays a critical role in some application domains and, therefore, should be taken into account. However, the existence of two tasks in the experiment poses a diff culty that has to be addressed. The time consumed to perform both tasks is not a good choice because the subject might fail giving an answer to one of the tasks and, as a consequence, the time measure would be invalid, giving an unrealistic perspective. So, instead of using the time used to perform both tasks, we measure time of the frst response which is the f rst keystroke; in other words, we measure the RT.

In total, there were $6 \times 30=180$ targets, and, as Table II shows, 65 empty responses in our experiments, which gives 115 valid time measurements. The summary statistics of the RT times are the following ones:

Min. 1st Qu. Median Mean 3rd Qu. Max. $\begin{array}{llllll}456.0 & 589.0 & 680.0 & 678.0 & 772.5 & 953.0\end{array}$

We should remark that these RTs are measured for each target, while the RTs shown in Table II are average values computed for each subject. The histogram in Fig. 3 completes the description of the RT showing that its distribution is quite symmetrical with its mean placed on $680 \mathrm{~ms}$. It is clear that there is a lower bound to the RT, which in our data is $456 \mathrm{~ms}$; the upper limit is imposed by the time window in which the subjects could provide the identif cation, in this case $1000 \mathrm{~ms}$.

The histogram in Fig. 3 shows the presence of a lower bound to the time required by the collective target identif cation. Collective identif cation requires at least this time in order to gather the individual responses. In order to better understand this relationship, we have plotted Fig. 4, which relates the accuracy and number of empty responses with the RT. To plot Fig. 4, we computed the accuracy of the collective identif cation with the responses given with a RT less than a value $t_{0}$. Then, this process was repeated for different values of $t_{0}$ between 200 and $1000 \mathrm{~ms}$.

As hypothesized, Fig. 4 shows that there are no answers until $450 \mathrm{~ms}$, and therefore, the accuracy before that moment is zero. After that, the subjects begin to provide answers (see the
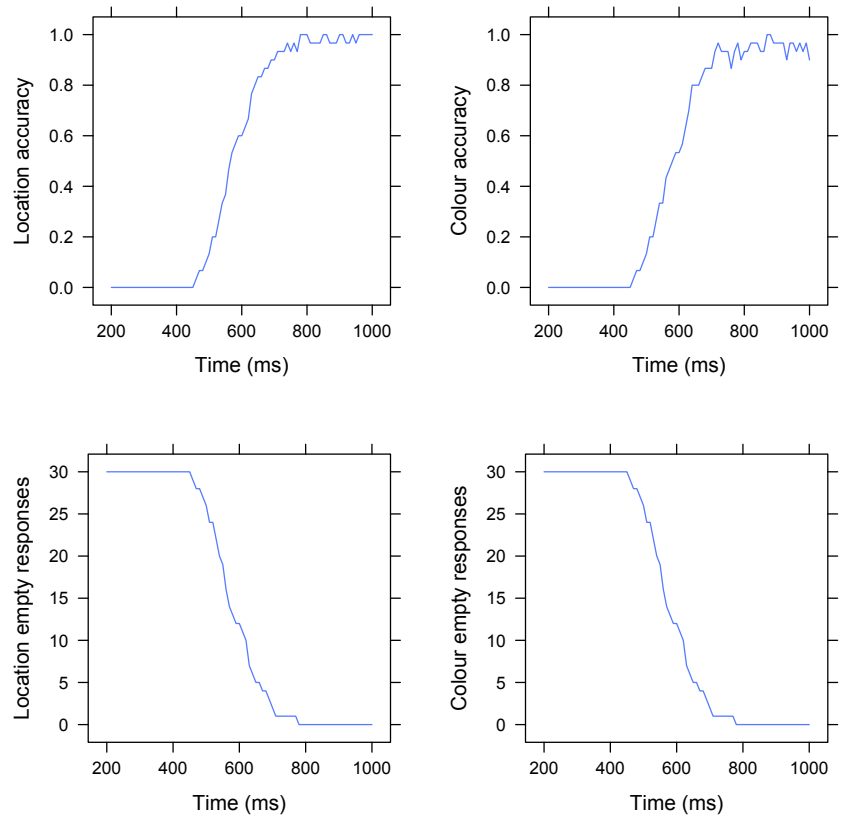

Fig. 4. Accuracy and empty responses of location identif cation (left column) and color identif cation (right column) as function of the time.

bottom row of Fig. 4) and subsequently the accuracy begins to increase with time, at a constant rate until a saturation point. In this point almost all the responses needed to identify the target accurately are available, but the new responses do not improve the performance. We also can observe some oscillations in the accuracy generated by the random choice done in case of draw.

Figure 4 also shows the presence of two boundaries in order to get an accurate identif cation with time constrains: A lower boundary given by the minimum RT and an upper boundary that limits the accuracy improvement. Between these two boundaries, there is a trade-off, a faster collective identif cation is feasible at a cost of a lower accuracy; and the opposite, a more accurate identif cation can be done if more time is allowed.

These experiments show that there are at least two factors that inf uence the identif cation accuracy: number of subjects and time window. However, these factors are not independent, a larger window time enables getting more responses. To better understand their relationship, we plotted the accuracy versus the number of subjects and time following the same procedure previously described; simply we have introduced both variables in the plot. The result is in Fig. 5.

Figure 5 relates the accuracy to the number of subjects and time. The behavior is as expected. The boundaries of the response time are clear in the f gure. The lower boundary remains constant regardless of the number of subjects involved in the identif cation; on the contrary, the upper boundary depends on the number of subjects, with limits, including that the involvement of more subjects decreases the time required to achieve a perfect accuracy, suggesting that we can achieve 


\section{Location accuracy}

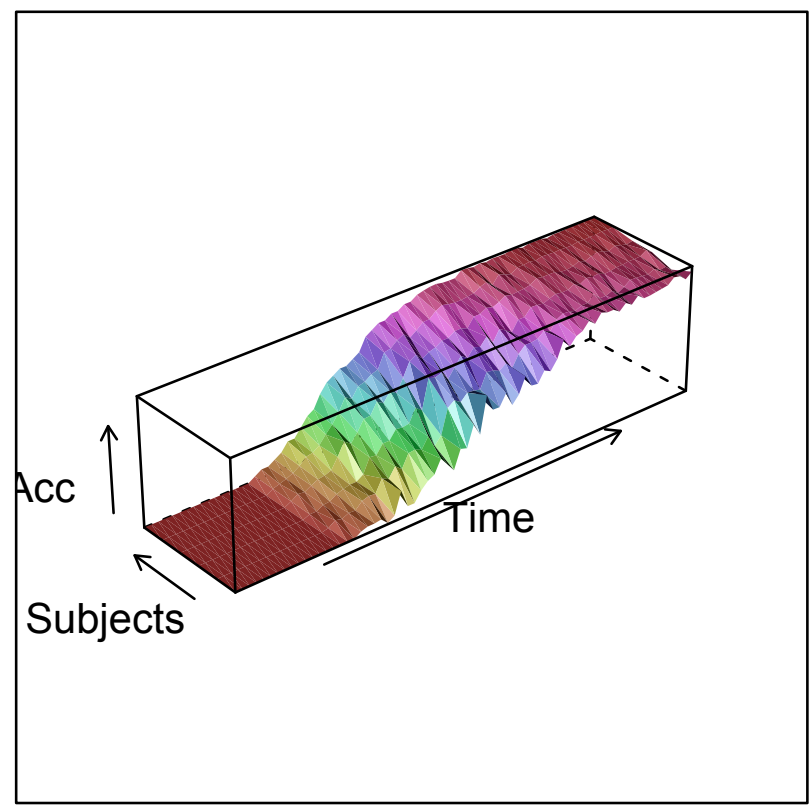

Colour accuracy

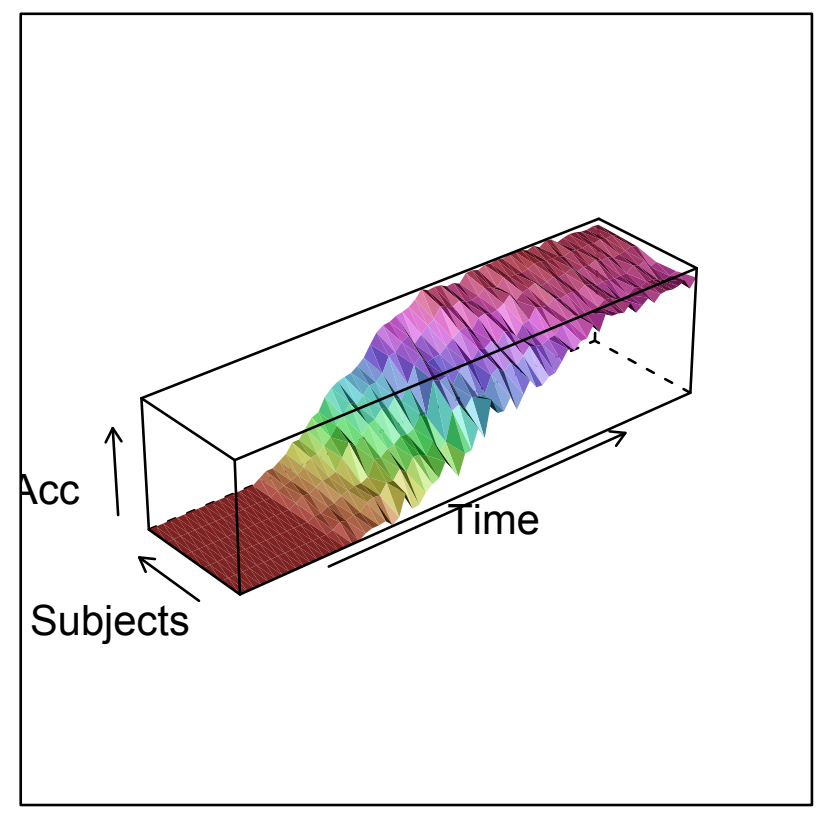

Fig. 5. Accuracy of location (left column) and color identif cation (right column) versus the time and number of subjects.

better identif cation accuracy in less time.

Based on these results, we conclude that there is a lower boundary of around $450 \mathrm{~ms}$ to the identif cation time given by the minimum time required by the users to perceive the target, process it mentally, and provide the mechanical response. Beyond a saturation point, providing more time does not increase the accuracy signif cantly. All these results have considered average values; in the following section we study the responses individually.

\section{FINE-GRAIN ANALYSIS}

In this section we aim to analyze the results individually, studying the identif cation accuracy depending on each target and subject. This will provide a fine-grain data that would allow us a deeper understanding of the results.

Figure 6 relates the median RT and the accuracy of each subject. We could expect a positive correlation between time and accuracy, those subjects that require more time should be able to identify better the target. However, our data do not support this hypothesis; on the contrary, it shows a lack of pattern in the data. This result contradicts the one reported by [14] showing a linear relationship between the accuracy and median RT. An explication to this discrepancy can be found in the mechanical part of our experiment. In opposition to [14], in our experiment the subjects had to provide a mechanical response, while [14] captures the ERP signal directly from the brain cortex. The lack of correlation between accuracy and reaction time observed in our experiment suggests that the mechanical response induces variability to the RT, probably
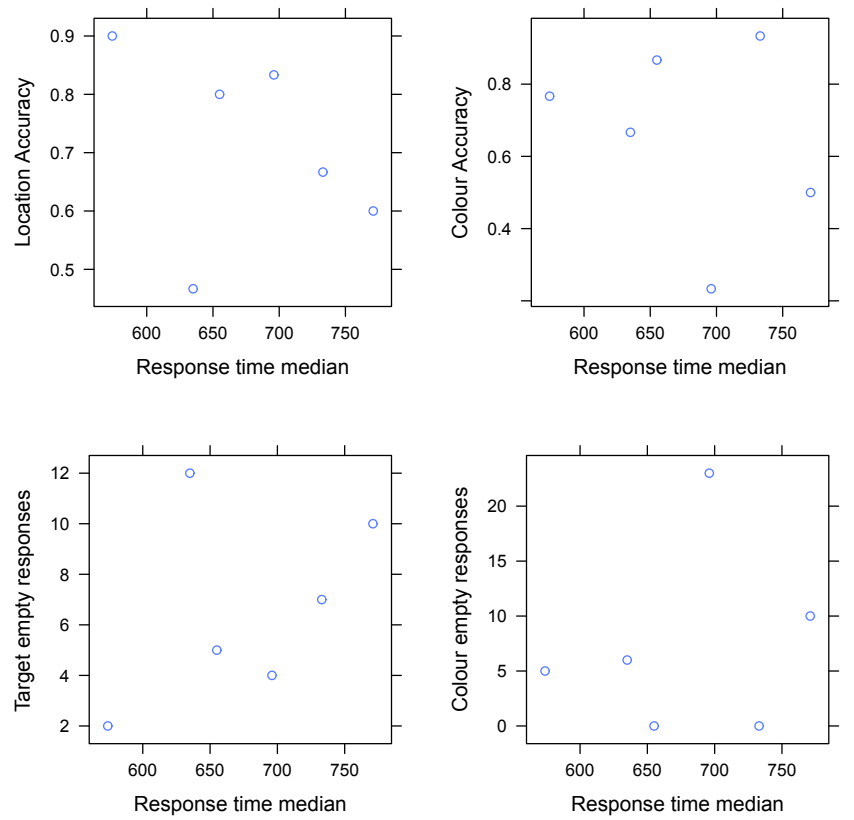

Fig. 6. Scatterplot relating the accuracy and median response time per subject. Each point in the plot represents one subject.

depending on the subject personal skills, for instance, if the subject performs any activity that requires mechanical coordination, such as playing piano or videogames. 

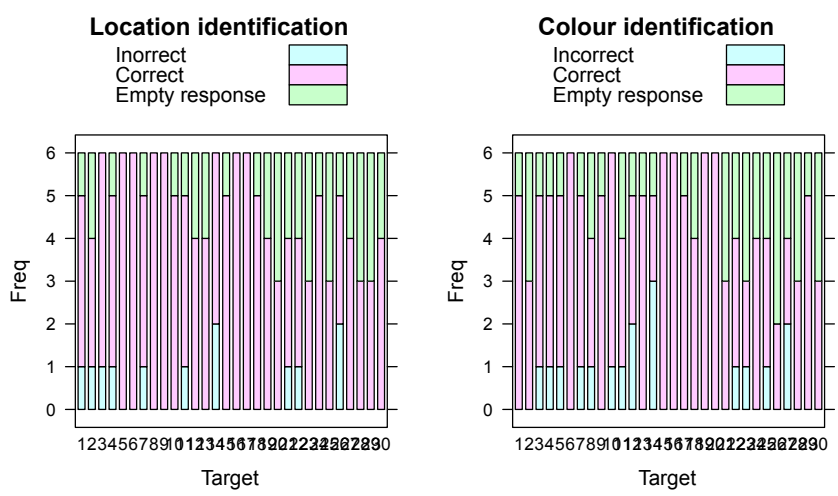

Fig. 7. Number of correct, incorrect and empty responses for location (left) and color (right) identif cation.

The result of the identif cation for each target is visualized in Fig. 7. Both tasks, the choice of location and color, give similar results: the loss of accuracy is the result of empty responses more so than the incorrect identif cations. In addition, the f gure suggests that the results get worse in targets presented towards the end; in particular, there are more empty responses there than in the beginning of the sequence. The probable cause is fatigue, or possibly a reduced excitement with the task. To avoid this, one may split the target series into two or three shorter ones.

\section{EEG ANALYSIS}

The final goal of this research is to substitute the input keyboard with a $\mathrm{BCI}$, using the non-invasive EEG signal acquired from the subject to identify the location and position of the target. This exciting topic has generated a great interest in the research community yielding, as a result, a large corpus of literature addressing this problem from different perspectives [15]. Perhaps, the best results comes from the detection of the P300 wave, also known as event related potential or simply ERP [16], [17]. Different techniques have been proposed, such as Genetic Programming [18] or Evolution Strategies [19].

In this preliminary study, we just recorded the brain activity of a subject performing the test previously described. The subject is a 35 years old male who performed the test sitting on a chair, in a low noise environment. The subject was requested to avoid any motion, with the exception of the eyes, that could be grazed into the target, to avoid any interference with the EEG. In order to perform a neuronal activity, the subject had to identify the color and mentally (silently) name it.

We used a commercial, low-cost, EPOC Emotiv device to acquire electrical signals from the head (while EMOTIV presents it as an EEG capturing device, the users tend to agree that the signals include also EMG, EOG, etc). EPOC has 14 active channels, and two reference channels and samples at a rate or $128 \mathrm{~Hz}$ in the band $0.2-45 \mathrm{~Hz}$ with a resolution of 16 bits. The data acquisition began with the test and lasted until its end, 30 seconds later.

Figure 8 plots the resulting EEG. There are vertical lines marking the beginning of a new target (700 ms stimulus).
Its color indicates the target color, which is green, yellow or red; green vertical lines indicate a $200 \mathrm{~ms}$ pause. The bad adherence of some electrodes introduced an unacceptable amount of noise to the signal in some channels, which were ignored. The $\mathrm{S} / \mathrm{N}$ ratio I poor, however, channel F4 clearly shows periodic peaks that, interestingly, coincide in time with the appearance of the target. This correlation appears along all the data sequence, as well as along all the sequence it correlates closely the emergence of the stimuli.

While this research is not yet completed, the magnitude of the peaks in channel F4 indicates, however, that it is unlikely to be EEG. As F4 is placed on the frontal bone, close to the eye, this gives credence to another explanation of the origin of this signal, which is extraocular or frontal muscles. To test this hypothesis, we can compare F4 to its symmetrical channel F3, but the result of this comparison is not conclusive. F3 presents much noise, and the peaks are not so clear, however, it seems to contain some peaks, for example, around $t=11 \mathrm{~s}$, but this is unclear so far. Another conjecture that perhaps could explain the F4 periodic signal is that the peaks had some connection with the heartbeats; this will be easily verif able in a future experiment, yet unfortunately current data recording stopped immediately after the last target, without any quiet period.

\section{CONCLUSIONS AND FUTURE WORK}

The experimental results reported in the paper show that the collaborative target location identif cation outperforms individual ones, even when the individual is skilled. There are additional benef ts from the collaborative approach: the number of empty responses decreases and it can provide reliable identif cations in less time. In addition, involving more than three people had no signif cant improving effect, which, however, it may be because the complexity of the task was low. In this case, this observation opens an opportunity to design faster target identif cation with keeping the accuracy. If more people are involved in the identif cation task, taking the frst three responses might lead to a fast and reliable identif cation.

The experiments also showed that the RT is limited by physiological constrains. In particular, the mechanical response required to the users requires a signif cant time, specially when taking into account that the brain only needs around $200 \mathrm{~ms}$ to react. The mechanical response takes, in average terms, around 600 milliseconds. A natural way to increase the RT, which is critical in certain applications, is to avoid the mechanical response by detecting and processing the brain activity.

The results of this study open new directions to increase the accuracy by means of more complex voting schemes, by exploiting, for instance, the best reliable subjects, or non-trivial correlations. The result of this study justif es further research along this line, in particular by employing more powerful EEG analysis techniques, and aggregating the signals from different brains to implement a Collaborative BCI.

This study has a number of limitations that should be emphasized. Firstly, the number of subjects (6) involved in the experiments is small and care should be used in drawing general conclusions. Though unclear if this may be the case, 


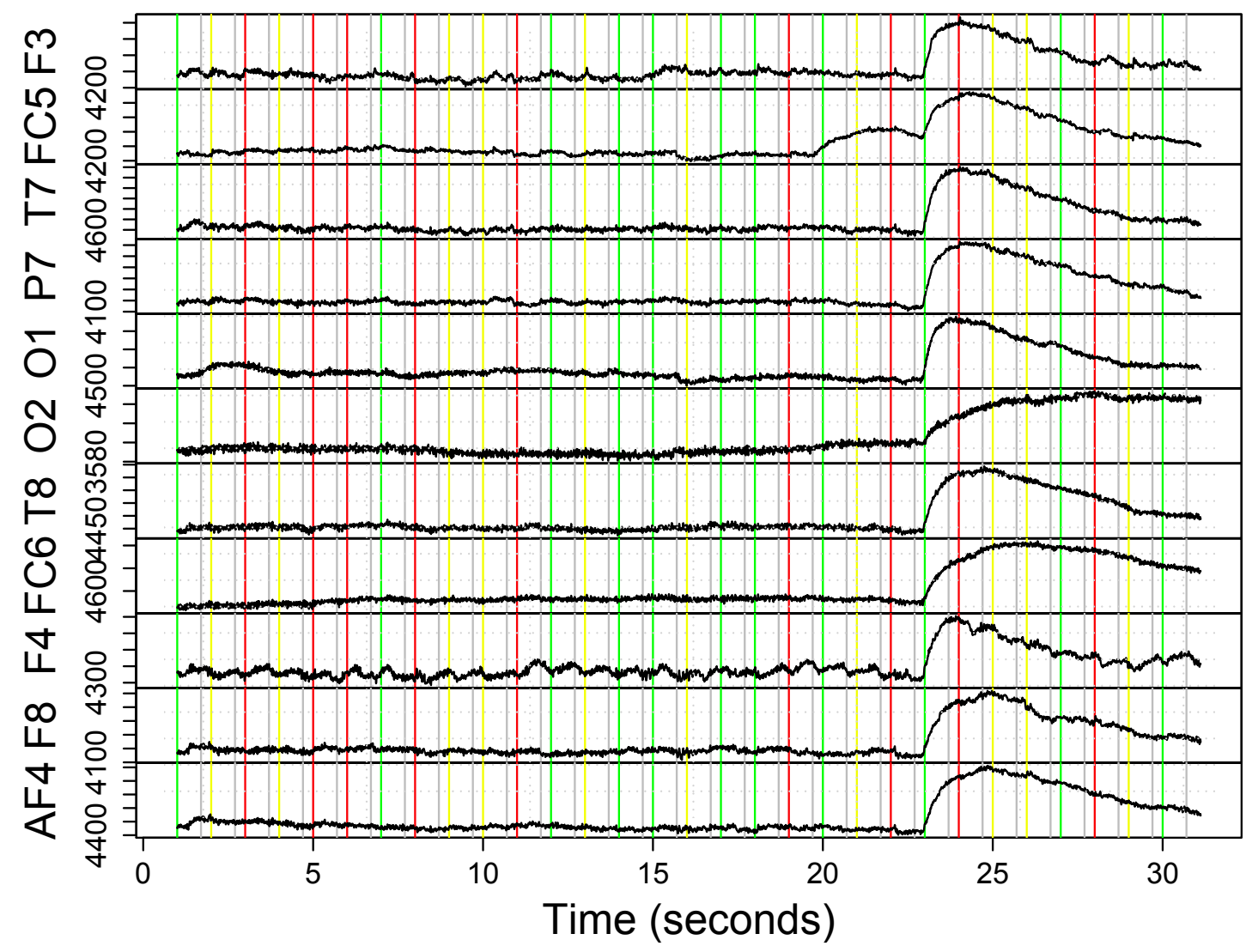

Fig. 8. EEG of a male, 35 years old, subject performing the target identif cation task. The arrival of a target is marked with a vertical line of the same color than the target (green, yellow or red), grey vertical lines correspond to pauses without target in the screen.

the selection of the subjects might also have biased the results, since they were relatively young subjects (around 30 years old), almost all were males (f ve out of six), and with a high educational level $(\mathrm{PhDs})$ in engineering. In addition, this study has not addressed important topics, such as potential correlations between the color and location responses, or the effects of a complex and stressful environment to subjects responses, all topics intended to be addressed in future research.

\section{ACKNOWLEDGMENT}

The research was carried out at the Jet Propulsion Laboratory, California Institute of Technology, under a contract with the National Aeronautics and Space Administration. Dr Barerro was supported by the University of Alcalá Mobility Grant. The authors gratefully acknowledge the support by the UK EPSRC Global Engagements grant EP/K004638/1.

\section{REFERENCES}

[1] Y. Wang and T.-P. Jung, "A collaborative brain-computer interface for improving human performance." PloS one, vol. 6, no. 5, p. e20422, Jan. 2011. [Online]. Available: http://dx.plos.org/10.1371/journal.pone. 0020422
[2] J. Millan, F. Renkens, J. Mourino, and W. Gerstner, "Noninvasive brain-actuated control of a mobile robot by human eeg," Biomedical Engineering, IEEE Transactions on, vol. 51, no. 6, pp. 1026-1033, june 2004.

[3] A. Stoica, "Aggregation of bio-signals from multiple individuals to achieve a collective outcome," Patent USPTO 20120203725,01 19, 2011.

[4] M. P. Eckstein, K. Das, B. T. Pham, M. F. Peterson, C. K. Abbey, J. L. Sy, and B. Giesbrecht, "Neural decoding of collective wisdom with multi-brain computing." NeuroImage, vol. 59, no. 1, pp. 94-108, Jan. 2012.

[5] A. Stoica, "Multimind: Multi-brain signal fusion to exceed the power of a single brain," in Emerging Security Technologies (EST), 2012 Third International Conference on, sept. 2012, pp. $94-98$.

[6] R. Poli, C. Cinel, F. Sepulveda, and A. Stoica, "Improving decisionmaking based on visual perception via a collaborative brain-computer interface," in 2013 IEEE International Multi-Disciplinary Conference on Cognitive Methods in Situation Awareness and Decision Support (CogSIMA), Feb 2013.

[7] P. R. Laughlin, E. C. Hatch, J. S. Silver, and L. Boh, "Groups perform better than the best individuals on letters-to-numbers problems: effects of group size." Journal of Personality and Social Psychology, vol. 90, no. 4, pp. 644-651, 2006.

[8] F. Galton, "Vox populi," Nature, no. 75, pp. 450-451, 1907.

[9] R. Sorkin, C. J. Hays, and R. West, "Signal-Detection Analysis of Group Decision Making," Psychological Review, vol. 108, pp. 183-203, 2001.

[10] R. Poli, C. Cinel, A. Matran-Fernandez, F. Sepulveda, and A. Stoica, 
"Towards cooperative brain-computer interfaces for space navigation," in International Conference on Intelligent User Interfaces, March 2013.

[11] L. Rossini, D. Izzo, and L. Summerer, "Brain-machine interfaces for space applications," in Engineering in Medicine and Biology Society, 2009. EMBC 2009. Annual International Conference of the IEEE, Sept., pp. $520-523$.

[12] R. Poli, C. Cinel, A. Matran-Fernandez, F. Sepulveda, and A. Stoica, "Some Steps towards Realtime Control of a Space-craft Simulator via a Brain-computer Interface," University of Essex, Tech. Rep. CES-525, October 2012.

[13] P. Yuan, Y. Wang, W. Hu, H. Xu, X. Gao, and S. Gao, "Study on an online collaborative bci to accelerate response to visual targets," in World Congress on Medical Physics and Biomedical Engineering, May 2012.

[14] S. Thorpe, D. Fize, and C. Marlot, "Speed of processing in the human visual system.” Nature, vol. 381, no. 6582, pp. 520-2, Jun. 1996.

[15] J. Becedas, "Brain-machine interfaces: Basis and advances," Systems, Man, and Cybernetics, Part C: Applications and Reviews, IEEE Transactions on, vol. 42, no. 6, pp. 825 -836, nov. 2012.

[16] R. M. Chapman and H. R. Bradon, "Evoked responses to numerical and non-numerical visual stimuli while problem solving," Nature, vol. 203, pp. 1155-1157, 1964.

[17] J. Polich, "Updating p300: an integrative theory of p3a and p3b." Clinical Neurophysiology, vol. 118, no. 10, pp. 21282148, 2007. [Online]. Available: http://www.pubmedcentral.nih.gov/ articlerender.fcgi? artid $=2715154 \&$ tool $=$ pmcentrez\&rendertype $=$ abstract

[18] R. Poli, M. Salvaris, and C. Cinel, "A genetic programming approach to the evolution of braincomputer interfaces for 2-d mousepointer control," Genetic Programming and Evolvable Machines, vol. 13, pp. 377-405, 2012.

[19] R. Aler, I. M. Galvn, and J. M. Valls, "Applying evolution strategies to preprocessing eeg signals for braincomputer interfaces," Information Sciences, vol. 215, no. 0, pp. $53-66,2012$. 